\title{
Amazonie joti
}

A propos du livre Nï Jotï aiye: Jkyo Jwainï. Libro comunitario jotï: historia, territorio y vida

Pascale de Robert

\section{(2) OpenEdition}

\section{Journals}

\section{Édition électronique}

URL : http://journals.openedition.org/ethnoecologie/6131

DOI : 10.4000/ethnoecologie.6131

ISSN : 2267-2419

Éditeur

Laboratoire Eco-anthropologie et Ethnobiologie

\section{Référence électronique}

Pascale de Robert, «Amazonie jotii », Revue d'ethnoécologie [En ligne], 17 | 2020, mis en ligne le 30 juin 2020, consulté le 16 juillet 2020. URL : http://journals.openedition.org/ethnoecologie/6131 ; DOI

https://doi.org/10.4000/ethnoecologie.6131

Ce document a été généré automatiquement le 16 juillet 2020.

\section{(c) (i) (3) $\Theta$}

Revue d'ethnoécologie est mis à disposition selon les termes de la licence Creative Commons Attribution - Pas d'Utilisation Commerciale - Pas de Modification 4.0 International. 


\section{Amazonie jotï}

A propos du livre Nỉ Jotï aiye: Jkyo Jwainï. Libro comunitario jotï:

historia, territorio y vida

\section{Pascale de Robert}

1 Zent E., Zent S., JuaeMölö A., Jono T., Liye G., Yolo A., Melomaja A., IkyekajaIjtë D., Yaluja M., Aubojkyo A., Bowijte J., Wajkemaja S., Jtute U., Juae I., Mölö A., Jliye U., Juae L., Jtitekya K., Olabu L., Amigoja A., Yolo K., Bowijte K., Tujuokaña R., Jono M., Liye M., Juae M., Jono N., Konojto M., Ijtë G., Jkujkyo A., Aubojkyo J., Juainkoa A. (+ 75 joti) 2019 Ni Jotï aiye: Jkyo Jwaini. Libro comunitario jotï: historia, territorio y vida. Ediciones IVIC / Irwin Andrew Porter Foundation, Acaté Amazon Conservation, Global Diversity Foundation. Caracas: Editorial Arte, 529 p. ISBN 978-980-261-197-3

Les ouvrages sur l'Amazonie signés par des auteurs amérindiens sont encore bien rares. Souvent élaborés par une ou deux personnes, parfois signés avec le nom d'une organisation autochtone ou d'un groupe ethnique, ils sont plus généralement l'œuvre d'auteurs non amérindiens, écrivains ou anthropologues. Le livre NÏ JOTÏ AIYE: JKYO JKWAINÏ. Libro comunitario jotï: historia, territorio y vida (NÏ JOTÏ AIYE: JKYO JKWAINÏ. Livre communautaire joti : histoire, territoire et vie), voudrait rompre avec cette "tradition » déjà mise à mal par de nombreuses publications plus ou moins récentes ${ }^{1}$. 
Figure 1 : Première de couverture de l'ouvrage

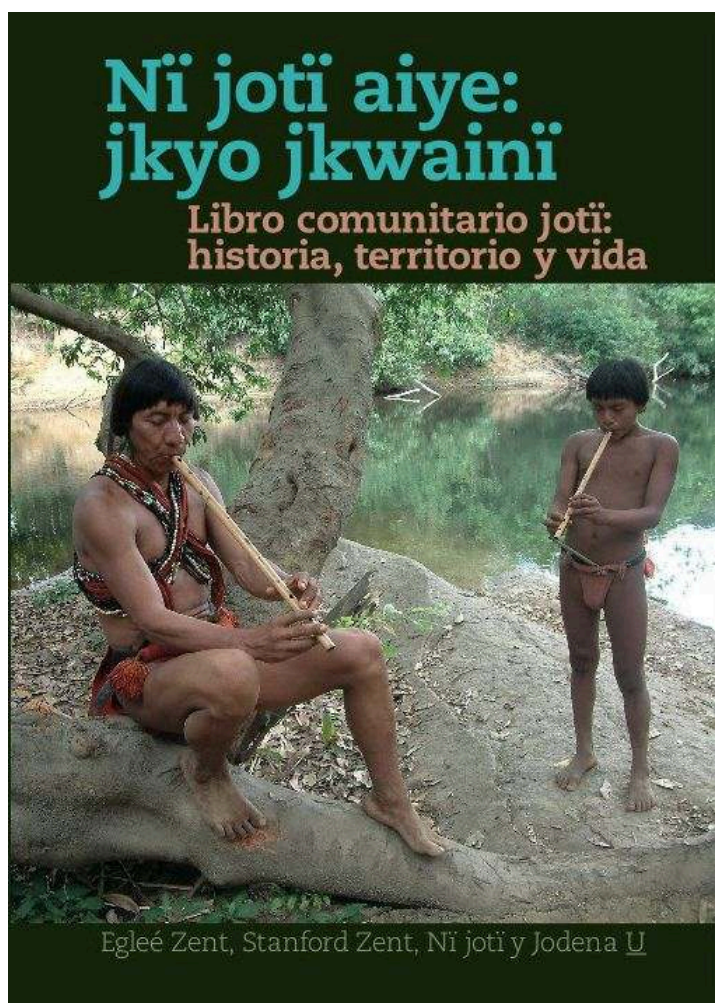

3 Comme nous le raconte son prologue, le titre du livre a été choisi - à l'instar du sommaire, des titres bilingues de chaque partie du texte, des thèmes développés dans chaque chapitre, des illustrations..., de façon collective au cours d'innombrables réunions, assemblées, rencontres et retrouvailles faites en langue jotï et impliquant au total un nombre significatif de personnes. Les auteurs donc, sont très nombreux : leurs noms remplissent d'ailleurs deux pages entières au début de cet ouvrage singulier. Et pourtant, en même temps, les auteurs sont bien peu nombreux: on évalue qu'environ 1700 personnes parlent et vivent jotï au sein de 25 villages de la région isolée des collines de Maigualida en Amazonie vénézuélienne. Une partie d'entre eux sont regroupés autour de deux missions religieuses, les autres dispersés dans la forêt. Les Jotï forment ainsi un tout petit peuple, bien différent de ses voisins, et occupent un territoire mal connu du reste du monde mais désormais très menacé, notamment par des projets d'exploitation minière qui se multiplient ici comme en bien d'autres endroits du bassin amazonien. 


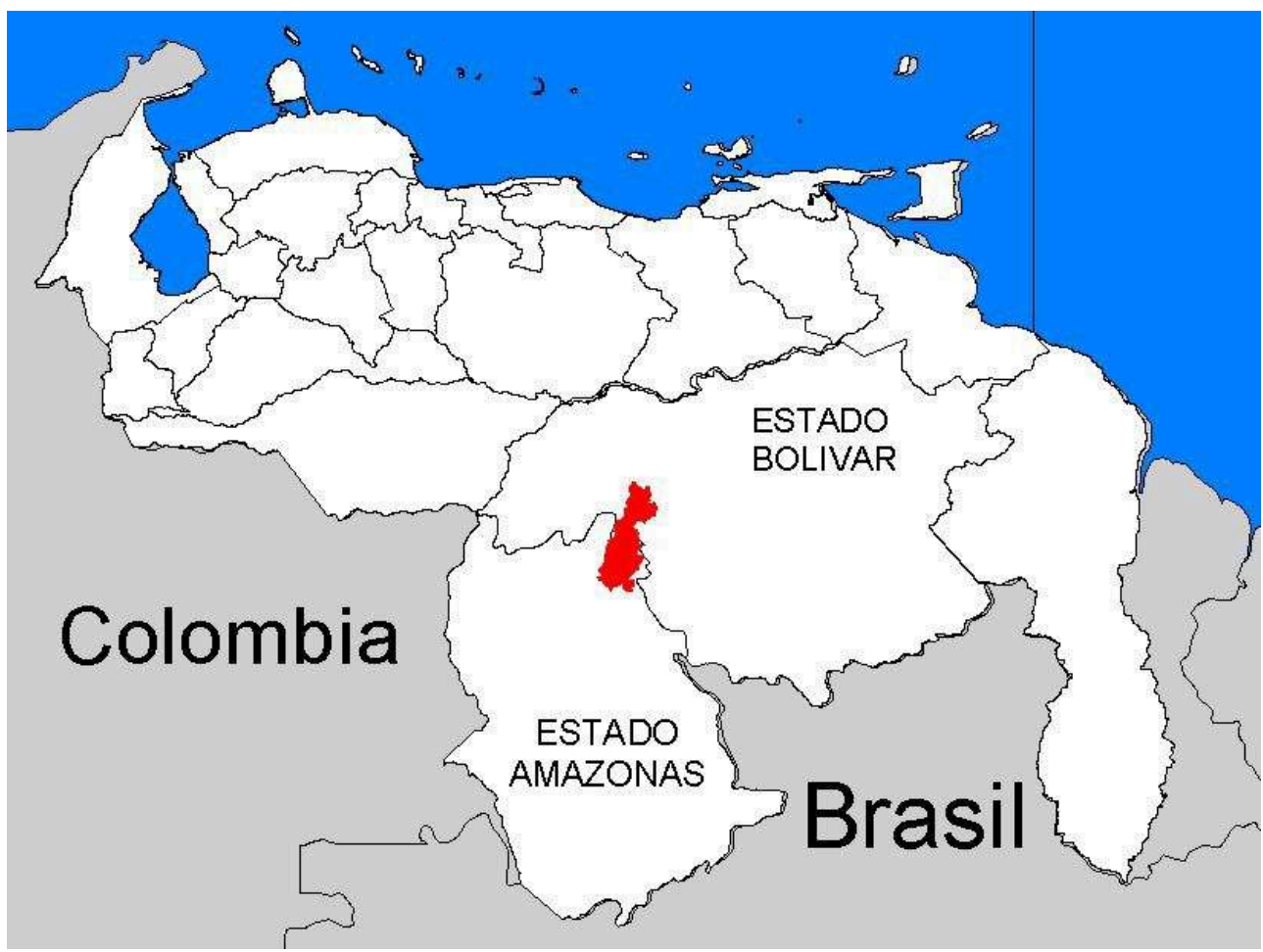

es menaces ont pesé lourd au moment de décider de se lancer dans l'aventure de création et de rédaction collectives comme laisse entendre le prologue. Cependant, et peut-être faut-il le regretter, le sommaire ne laisse rien transparaitre des violences et des drames socioculturels, territoriaux, écologiques et sanitaires qui marquent le quotidien de la plupart des communautés autochtones d'Amazonie. Loin de là, les Jotï semblent ignorer les exactions dont ils font l'objet, sans omettre non plus d'y faire allusion (comme l'orpaillage pour clore le troisième chapitre), mais sans insistance, comme s'il s'agissait d'événements secondaires à l'heure de parler de sa propre histoire. Tout se passe comme si les auteurs jotï avaient choisi de nous parler d'euxmêmes et de ce qui leur importe en laissant pour une fois dans l'invisibilité ceux qui s'acharnent, d'une toute autre manière, à les rendre invisibles.

Ce livre donc est celui des Jotï parlant des Joti.

6 Il faut dire quand même que les deux premiers auteurs ne sont pas nés dans un hamac puis honorés de la cérémonie des colliers comme tout Jotii ${ }^{2}$ qui se respecte. Ils ont été conviés à participer pour écrire «en espagnol, dans un langage accessible pour être utilisé par les générations actuelles et futures, dans les écoles...» (p. 25). Eglée Lopez Zent et Stanford Zent sont deux anthropologues de l'IVIC (Instituto Venezolano de Investigación Científica), institution de recherche renommée et éditrice de l'ouvrage. Spécialistes des Jotï avec lesquels ils travaillent depuis près de 25 ans, ils sont les auteurs de nombreux articles scientifiques importants pour la discipline, et en particulier pour l'ethnoécologie. Mais les lecteurs ne trouveront pas de références à leurs travaux en lisant, pas non plus à ceux des collègues et spécialistes de cette région du monde, puisque les Jotï ont décidé, sauf exception, de ne pas faire mention des études les concernant de près ou de loin dans le cours du texte (des références bibliographiques spécialisées sont regroupées en fin d'ouvrage). En revanche, le livre est truffé de citations qui rapportent les histoires racontées par les Amérindiens et 
s'enchainent par thèmes avec, pour chacune, la mention du nom du locuteur/auteur, d'une date et souvent d'un lieu. Beaucoup de pages se présentent comme un patchwork de phrases dites et entendues, transcrites et traduites, puis cousues ensemble en forme d'un discours continu, d'un murmure. Cette option, parmi d'autres, ouvre le chemin à une éthique et de nouvelles pratiques de recherche collaborative en questionnant, dans le même temps, nos catégories et modes d'écriture. Ainsi par exemple, même si l'ouvrage est organisé en chapitres qui semblent annoncer une monographie ethnographique classique, on se retrouve parfois à lire, selon les endroits, un essai philosophique, un traité de botanique, un roman ou un poème.

7 Chacun des neuf chapitres annonce un thème en langue jotï puis espagnole. Après le prologue présentant les modalités et les enjeux de la démarche, le deuxième chapitre aborde la vaste question du « qui sommes nous, nous les gens véritables? » et rapporte la mémoire des premiers contacts établis, à partir des années 70 , avec ceux qui portent des vêtements, et des maladies (grippe et malaria). Le troisième chapitre Jkyo nous fait connaître le territoire des Jotï avec les revendications qu'ils ont portées au gouvernement de la République bolivarienne du Venezuela. On peut écouter ensuite comment "trois fois la terre a été créée et deux fois détruite» et autres mythes racontés par les plus anciens, transcris et traduits sans analyse mais accompagnés d'éléments introductifs à des concepts-clé (terre, personne...), pour aborder notamment les relations entre collectifs humains et non-humains des temps mythiques jusqu'à aujourd'hui. Les chapitres suivants traitent respectivement des savoirs et savoir-faire écologiques des Jotï, des fêtes et pratiques rituelles qui ponctuent la vie des personnes, de la médecine traditionnelle avec les conceptions du corps et des maladies. Après nous avoir conduit, pendant plus de 400 pages, dans les sphères de tous ces savoirs, les Jotï nous introduisent, dans les deux derniers chapitres, à la philosophie jkyojkwaini qui est une attention bienveillante à la vie et à tous les êtres animés et inanimés, visibles et invisibles, qui peuplent la forêt. Avec elle, ils posent encore des questions actuelles, comme l'impact des missions ou de l'école avec la sédentarisation, et nous disent « au revoir », comme à la fin d'une fête.

8 Cet ouvrage encyclopédique restera une référence au moins pour les Jotï et pour l'anthropologie des basses terres. Signalons qu'il comporte également une iconographie très belle et fournie (dessins, cartes, schémas, nombreuses photographies anciennes et récentes). Mais parmi tous les sujets qui mériteraient d'être mentionnés, quelques-uns semblent plus particulièrement intéressants pour les lecteurs de la Revue d'Ethnoécologie.

9 La publication des cartes du territoire jotï, et le récit des étapes de leur élaboration dans le cadre du processus d'auto-démarcation des territoires amérindiens, est importante. En dépit d'une situation sanitaire, socio-environnementale et politique défavorable en ces temps difficiles, la reconnaissance du travail cartographique fait par les Jotï est un premier pas vers l'application des droits constitutionnels, la République bolivarienne du Venezuela reconnaissant aux peuples autochtones des droits spécifiques sur leurs territoires traditionnels. La publication de cette aventure collective vient ainsi combler un peu le manque de connaissances sur l'histoire et la géographie d'une région habitée, nommée et cultivée (dans les deux sens du terme) par les Jotï depuis des temps immémoriaux. Elle adopte une perspective semblable à d'autres expériences menées un peu partout en Amazonie, où l'on constate, y compris en Guyane française, un recul des droits des peuples autochtones et une avancée des 
menaces sur la forêt et ceux qui l'habitent (invasion de terres, exploitation illégale, implantation de méga-projets miniers ou hydroélectriques) ${ }^{3}$. Pour cette raison, on peut regretter que l'ouvrage ne puisse donner plus de détails sur la situation actuelle du territoire jotï, comme le choix de ne pas insister plus sur les enjeux politiques de la cartographie ou de questions légales (difficulté de prouver sa nationalité p.115, consentement préalable, droit des peuples indigènes) ${ }^{4}$.

Le Livre communautaire jotï apporte également des éléments pour les discussions actuelles en anthropologie sociale sur les processus de patrimonialisation et de transmission, l'écologie historique en Amazonie, le thème de la domestication (végétale en particulier) ou les ontologies amérindiennes. Surtout, c'est une œuvre majeure d'écologie jotï qui détaille de façon approfondie les savoirs amérindiens sur des écosystèmes très complexes, mal connus et caractérisés par une très haute biodiversité. Les connaissances des plantes ${ }^{5}$, centrales pour les Jotï, médicinales et autres, mais aussi les techniques de collecte du miel ou des champignons, les outils de pêche et des pratiques horticoles spécifiques font l'objet de descriptions détaillées sur lesquelles devraient s'attarder tous ceux qui s'intéressent aux ethnosciences. Au fil des pages, ces manières de cultiver, de se soigner ou de chasser nous mènent sur les chemins jotï, une singulière manière d'être au monde, de penser la vie et l'échange.

11 «Je rêve d'un seul récit

qui dicterait sans faute

toute une vie vécue $»^{6}$.

Pour terminer, je voudrais insister sur l'intérêt littéraire de l'ouvrage, même si celui-ci n'était sans doute pas prioritaire pour les auteurs, ni pour les éditeurs. Le choix de transcrire les récits de personnes très différentes (enfants, chamans, leader...) citées les unes après les autres, d'intercaler ou pas des commentaires et des analyses, et d'utiliser les mots de leur langue chaque fois qu'une traduction en espagnol semblait trop approximative, était un vrai défi. En dépit de la juxtaposition de styles d'écriture différents (listes, confidences, descriptions, dialogues, récits de vie et de voyage...) et de l'abondance de noms propres et de noms communs jotï qui ne sont pas distingués par une graphie en italique, la lecture n'est pas vraiment difficile. Il faut croire que les auteurs ont su transmettre la multiplicité de leurs voix, même présentées en « un seul récit », et un peu de l'oralité amérindienne dans cet exercice de littérature, peut-être malgré eux. Ainsi, au terme de deux ou trois chapitres peut-être, le lecteur se sent pris dans un rythme particulier, une musique, tant et si bien qu'il peut avoir l'illusion, à certains détours du livre, qu'il comprend le jotï.

\section{BIBLIOGRAPHIE}

Bacon J. \& Acquelin J. 2011 - Nous sommes tous des sauvages. Montréal, Mémoire de l'Encrier, 70 p.

Kopenawa D. \& Albert B. 2013 - La chute du ciel. Paroles d'un chaman yanomami. Paris, Plon, 828 p. (Terre humaine). 
Krenak A. 2019 - Ideias para adiar o fim do mundo. São Paulo, Companhia das Letras, 64 p.

Lana F.A. \& Lana L.G. 1995 - Antes o mundo não existia: Mitologia dos antigos Desana-Kehíripõrã: Umusi Pãrõkumu e Tõrãmu Kehíri. São Gabriel da Cachoeira, UNIRT/FOIRN ed., 240 p.

Levis C. et al. 2017 - Persistent effects of pre-Columbian plant domestication on Amazonian forest composition. Science 355 (6328) : 925-931.

Thiérion B. 2018 - Émergence d'une littérature autochtone au Brésil : Daniel Munduruku, un écrivain aux multiples facettes. In : Côté J.-F. (Ed.) La renaissance des cultures autochtones: enjeux et défis de la reconnaissance. Québec, Presses de l'Université Laval : 95-119.

Zent E. \& Zent S. 2004 - Los Jodï: sabios botánicos del Amazonas venezolano. Antropológica 97 (98) : 29-70.

Zent S. \& Zent E. 2012 - Jodï horticultural belief, knowledge and practice: incipient or integral cultivation?. Boletim du Museu Paraense Emilio Goeldi 7 (2) : 293-338.

Zent S., Zent E.L., Mölö L.J. \& Chonokó P. 2016 - Reflexiones sobre el proyecto Auto-Demarcación y EtnoCartografía de las Tierras y Hábitats Jodï y Eñepa. Revue d'ethnoécologie (9) [En ligne] URL : http://journals.openedition.org/ethnoecologie/2670.

\section{NOTES}

1. Il est temps que les auteurs originaires des peuples dits sans écriture entrent en littérature autrement qu'en héros de biographie ou de sagas exotiques. Citons quelques entreprises, parmi d'autres, qui ont déjà marqué "l'émergence de voix autochtones dans le champ littéraire " (Thiérion 1998) de genres divers: la collection Narradores Indígenas do Rio Negro (NIRN) avec l'ouvrage précurseur de Firmiano et Luiz Lana "Avant, le monde n'existait pas ", les Ediçoes Ikôré, les ouvrages de Daniel Munduruku, les essais de Ailton Krenak, «La chute du ciel » écrite en binôme par un chamane et un anthropologue amis inséparables depuis des décennies, ou, pour sortir du Brésil mais rester en terre amérindienne, les poèmes innu de Joséphine Bacon au Québec.

2. Un terme qui se traduit par « les gens » dans leur langue.

3. À ce sujet on peut consulter les dossiers Cartographie participative de cette même revue où les aspects méthodologiques de l'expérience jotï ont déjà fait l'objet d'analyse.

4. Rappelons que les anthropologues sont bien souvent susceptibles de censure. Certaines informations complémentaires sont disponibles en ligne : https://www.globalenvironments.org/ alleviating-malaria-in-venezuela/ et https://watanibasocioambiental.org

5. Les listes des noms de plantes jotï avec leurs correspondants, quand c'est possible, en espagnol et latin sont en annexe (environ 250 entrées), comme les noms de poissons, et d'animaux. Au delà des usages et principes de catégorisation des plantes discutés dans le livre, Zent \& Zent (2004, 2012) ont publié sur l'ethnobotanique et l'agriculture jotï, en écologie historique (Levis et al. 2017), entre autres travaux.

6. Extrait d'un poème de Joséphine Bacon, dans l'ouvrage « Nous sommes tous des sauvages » (2011) 